\title{
Permittivity and Tan Delta Characteristics of Epoxy Nanocomposites in the Frequency Range of $1 \mathrm{MHz}-1 \mathrm{GHz}$
}

\author{
Santanu Singha and M. Joy Thomas \\ High Voltage Laboratory \\ Department of Electrical Engineering \\ Indian Institute of Science \\ Bangalore, 560 012, India
}

\begin{abstract}
To achieve a compact and reliable design of electrical equipment for the present day requirements, there is an urgent need for better and smart insulating materials and in this respect, the reported enhancements in dielectric properties obtained for polymer nanocomposites seems to be very encouraging. To further understand the dielectric behavior of polymer nanocomposites, this experimental work reports the trends of dielectric permittivities and tan delta (loss tangent) of epoxy nanocomposites with single nano-fillers of $\mathrm{Al}_{2} \mathrm{O}_{3}$ and $\mathrm{TiO}_{2}$ at low filler concentrations $(0.1 \%, 0.5 \%, 1 \%$ \& $5 \%)$ over a frequency range of $1 \mathrm{MHz}-1 \mathrm{GHz}$. Results show that the nanocomposites demonstrate some very different dielectric characteristics when compared to those for polymer microcomposites. Unlike the usual expectations of increasing permittivity with increasing filler concentration in polymer microcomposites, it has been seen that up to a certain nano-filler concentration and depending on the permittivity of the nano-filler, the permittivities of the epoxy nanocomposites are less than that of the unfilled epoxy at all the measured frequencies. This suggests that there is a very strong dependence of the filler concentration and nano-filler permittivity on the final permittivity of the nanocomposites at all these frequencies. But, in the case of tan delta behavior in nanocomposites, significant effects of filler concentrations were not observed with both $\mathrm{Al}_{2} \mathrm{O}_{3}$ and $\mathrm{TiO}_{2}$ fillers. Tan delta values in nanocomposites with $\mathrm{Al}_{2} \mathrm{O}_{3}$ fillers are found to be marginally lower at all filler concentrations when compared with the value for unfilled epoxy. But, in $\mathrm{TiO}_{2}$-epoxy nanocomposites, although the variations in tan delta are not significant with respect to unfilled epoxy, some interesting trends are observed with respect to the frequencies of measurement.
\end{abstract}

Index Terms - Nanocomposites, nanodielectrics, permittivity, tan delta, epoxy.

\section{INTRODUCTION}

POLYMER nanocomposites with better dielectric and electrical insulation properties are slowly emerging as excellent functional materials for dielectrics and electrical insulation application and the term "nanodielectrics" for such materials is increasingly becoming popular. Although the technology of addition of fillers to polymers to enhance a particular dielectric property has been in existence for several decades [1-3], the effect of filler size on the dielectric property of the polymer composites has not been understood fully. It is with the advent of nanotechnology leading to the availability and commercialization of nanoparticles that polymer nanocomposite technology started to gain momentum. Polymer nanocomposites have been found to exhibit enhanced physical, thermal and mechanical properties when compared to the traditional polymer materials and that too at low nano-filler concentrations (1-10\%) [4-6]. But it is only

Manuscript received on 7 December 2006, in final form 9 August 2007. recently that the dielectric properties of such polymer nanocomposites were looked into and limited research results demonstrate very encouraging dielectric properties for these materials. Irrespective of the type of base polymer material (thermoplastic or thermoset), significant enhancements in several physical properties, like thermal conductivity (with conducting fillers) or dielectric properties like resistivity, permittivity, dielectric strength, tracking and partial discharge resistant characteristics (with insulating fillers) were observed when compared to similar properties in traditional polymer microcomposites [7-9]. These observations were mainly attributed to the unique properties of nanoparticles and the large interfacial area in polymer nanocomposites [10-12].

The present work looks into two of the dielectric properties in epoxy nanocomposites - permittivity and tan delta. Permittivity determines the charge storage capacity of a dielectric material as well as dictates the electric field distribution in a composite insulation system whereas tan delta values indicate the dielectric losses possible in an insulating material. For any electrical 
insulation system, a low tan delta value is always desired in the dielectric material whereas the desired permittivity of the material can be high or low depending on the end application. To tune the values of permittivity and tan delta, polymer microcomposites were studied earlier and sufficient work on the variations of permittivity and tan delta with respect to frequency and temperature are available [13-17], but again these studies are mostly with conducting fillers. As for polymer nanocomposites, it is only recently that some reports of permittivity and tan delta measurements have become available [18-23]. But, most of the reported investigations were performed with nano-filler concentrations higher than $5 \%$ by weight in the polymer matrix $[7,8]$ and information on the dielectric properties of polymer nanocomposites with low filler concentrations (at the sub 5\% level) are very scarce in literature. Investigations into the mechanical properties of polymer nanocomposites have reported significant improvements with very low nano-filler concentrations [24]. Since the same nanocomposite systems have to perform dielectric functions also in many applications, significant variations in the dielectric properties could be expected at low filler concentrations. Apart from the filler concentrations, a majority of the permittivity and tan delta measurements in nanocomposites with insulating or semiconducting fillers were performed from low frequencies up to $1 \mathrm{MHz}$ or few tens of $\mathrm{MHz}$. In studies where dielectric measurements of polymer nanocomposites were carried out in the higher or microwave frequency range, the fillers used were of the conducting type [18-23]. For polymer nanocomposites with insulating fillers, an understanding of the permittivity and tan delta behavior at frequencies higher than $1 \mathrm{MHz}$ is limited at present. Based on these facts, the current experimental investigation attempts to understand the behavior of dielectric permittivity and loss tangent in epoxy nanocomposites with $\mathrm{Al}_{2} \mathrm{O}_{3}$ and $\mathrm{TiO}_{2}$ nano-fillers at very low filler concentrations and at frequencies higher than $1 \mathrm{MHz}$.

\section{EXPERIMENTAL DETAILS}

\subsection{MATERIALS}

Epoxy, one of the most commonly used insulating materials in the electrical industry is chosen as the base polymer material for the current study. Bisphenol-A epoxy resin (CY1300) along with a Triethylene Tetramine hardener (HY956) of Huntsman make was used for the investigations. As for the nano-fillers, $\mathrm{Al}_{2} \mathrm{O}_{3}$ and $\mathrm{TiO}_{2}$ were chosen since they are well known materials with excellent electrical properties. Commercially available $\mathrm{Al}_{2} \mathrm{O}_{3}$ and $\mathrm{TiO}_{2}$ nanoparticles with average particle sizes of $45 \mathrm{~nm}$ and $50 \mathrm{~nm}$ respectively and procured from Sigma Aldrich were used as the fillers.

\subsection{NANOCOMPOSITE SAMPLE PREPARATION}

Nanodielectrics open a new window of opportunity for the electrical industry, but before they can be used in any specific applications, their properties have to be characterized accurately and one of the factors directly affecting the properties is the processing methodology of preparing nanodielectrics. Nanoparticles are highly charged materials which tend to agglomerate and form large particle sizes.
Nanoparticle agglomerations in a polymer matrix reduce the nanoparticle surface area to volume ratio significantly which in turn compromises the unique nanoparticle properties in the polymer. Therefore, proper processing techniques have to be adopted to ensure uniform dispersion of the nanoparticles in the polymer matrix. In the current study, nanocomposites were prepared using a combination of two different processing techniques - mechanical mixing and ultrasonication. These processing techniques were preferred in the current study because they are easy to use at the laboratory scale.

In the mechanical mixing method, the particles are mixed with the epoxy resin in a high shear mechanical mixer at a speed of $700 \mathrm{rpm}$ (revolutions per minute). The mixing time was decided based on the volume of resin/particle mix and is important since the polymer may tend to degrade if mixing is carried out for a longer duration. In ultrasonication technique, the inorganic fillers are mixed with the epoxy resin under normal hand stirring and then sonication is carried out in a water bath at a frequency of $24 \mathrm{kHz}$ with intermittent stirring.

An important parameter during the experiments is the need for vacuum evacuation during polymer processing. The presence of air bubbles, moisture or other foreign matter in the polymer matrix can act as defects, which in turn can drastically influence the dielectric properties of the epoxy composites. To remove any chances of air bubbles influencing the dielectric measurements, degassing of the polymer mix was carried out whenever required before the curing process of the resin-particle-hardener mix.

Initially, the epoxy resin and hardener are taken in two different beakers and they are both degassed at $40{ }^{\circ} \mathrm{C}$ for $2 \mathrm{~h}$. Similarly, the $\mathrm{TiO}_{2}$ particles were vacuum dried at $90{ }^{\circ} \mathrm{C}$ for $24 \mathrm{~h}$ before mixing it to epoxy. Approximately $40 \mathrm{ml}$ of resin is then poured into the mixer immediately and again degassing is carried out. Then, the required quantity of filler particles (based on weight fractions) is slowly dispersed into the epoxy resin with continuous hand stirring. The mechanical mixer was then operated at $700 \mathrm{rpm}$ for $90 \mathrm{~s}$. The resin-particle mix is then degassed till the air bubbles stop coming out of the mix (around 5 minutes due to the reduced viscosity of the mixture). Immediately after this degassing, $7 \mathrm{ml}$ of mix (required for one sample preparation) was poured into a different small beaker. For the ultrasonication process after mechanical mixing, the beaker is sonicated for 60 minutes. Then, the appropriate amount of hardener is poured into the beaker, mixed vigorously for few minutes and poured into the mold. The mold with the composite material is again degassed (to remove the air bubbles formed during hardener mixing) till the air bubbles stop coming out of the material. The mold is then left for curing inside an oven at $60{ }^{\circ} \mathrm{C}$ for $4 \mathrm{~h}$ (based on the epoxy specifications data sheet). Samples of $75 \mathrm{~mm}$ diameter and $1 \mathrm{~mm}$ thickness were molded and then they are kept under desiccation. Figure 1 shows representative microstructure images of cross-sections in epoxy nanocomposites for different filler concentrations. It can be seen from the SEM images that the nanoparticles are dispersed quite uniformly in the polymer matrix confirming that the two-step processing method utilized to prepare samples in the present study is very effective [25]. 


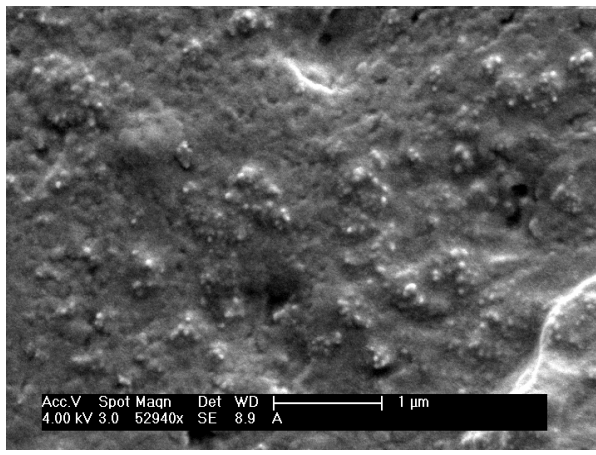

(a) $10 \%$ nano $\mathrm{TiO}_{2}$ by weight

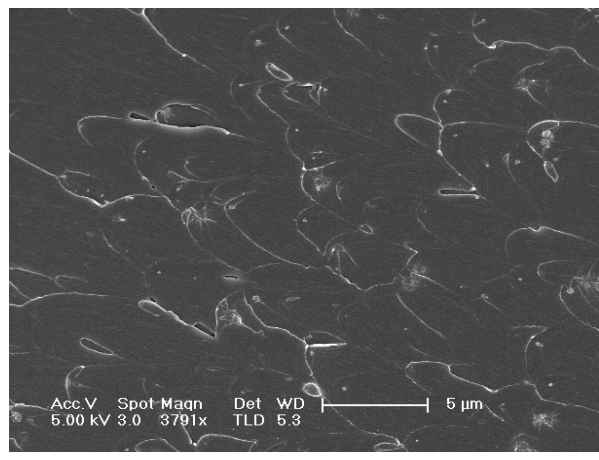

(b) $1 \%$ nano $\mathrm{TiO}_{2}$ by weight

Figure 1. Representative SEM microstructures showing the dispersion of $\mathrm{TiO}_{2}$ nanoparticles in the epoxy matrix.

\subsection{DIELECTRIC PERMITTIVITY AND TAN DELTA MEASUREMENTS}

An Agilent Impedance/Material Analyzer (4291B) with the appropriate dielectric test fixture was used to measure the dielectric permittivity and tan delta of the nanocomposites over the frequency range of $1 \mathrm{MHz}-1 \mathrm{GHz}$. Nanocomposite samples were prepared at least $24 \mathrm{~h}$ prior to the experiments and they are kept under vacuum evacuation after preparation. The accuracy of the impedance analyzer was confirmed by measuring the permittivity and tan delta (in the frequency range of $1 \mathrm{MHz}-1 \mathrm{GHz}$ ) of a standard PTFE sample provided by the manufacturers. The permittivity and tan delta data presented in this paper are an average value for 7 samples (with a deviation within $2 \%$ ) for a specific filler concentration. The samples were randomly picked up from different batches of preparations spread over several days. All the measurements were performed at a temperature of $27{ }^{0} \mathrm{C}$ and at a relative humidity of $60 \%$.

\subsection{DSC MEASUREMENTS}

The glass transition temperatures $\left(\mathrm{T}_{\mathrm{g}}\right)$ of the epoxy nanocomposites with $\mathrm{TiO}_{2}$ and $\mathrm{Al}_{2} \mathrm{O}_{3}$ fillers were measured to understand their thermal behaviors. The measurements were performed using a Dielectric Scanning Calorimetry (DSC) equipment of Mettler make. The rate of rise of temperature was set at $5{ }^{0} \mathrm{C}$ per minute and the measurements were performed up to $200{ }^{\circ} \mathrm{C}$. Data reported in this paper are for an average (deviation within 1\%) of 3 measurements with samples from different batches.

\section{RESULTS AND DISCUSSIONS}

The behavior of dielectric properties as a function of frequency in insulating materials (including composites) is governed by dielectric polarization and relaxation effects. The polarization phenomena in a dielectric material over a wide time scale can be seen in Figure 2 and it can be observed that different polarization mechanisms dominate over different frequency ranges. In a typical polymeric system based on an epoxy resin cured with an amine hardener as in the present case, permittivity and loss tangent are governed by the number of orientable dipoles present in the system and their ability to orient under an applied electric field of certain frequency [26, 27]. The dielectric relaxation mechanisms in an epoxy system at different curing temperatures, different extents of curing and different measurement frequencies can be grouped into three classes, viz. $\alpha, \beta$ and $\gamma$ relaxations $[28,29,30]$. $\alpha$ relaxation usually occurs at low frequencies when all the dipoles in the polymer can orient and relaxation of whole molecules take place, whereas $\beta$ and $\gamma$ relaxations occur at high frequencies mainly due to the relaxations caused by the motion of hydroxyl $(\mathrm{OH})$ groups in the polymer chain, motion of unreacted dipolar groups or due to motion of dipoles associated with other smaller molecular groups formed during the chemical reaction of the resin and hardener. Usually, in cured epoxy systems, the molecular groups attached perpendicular to the longitudinal polymer chain contribute to the dielectric relaxations since the effects of polarization due to the parallel dipolar groups in the longitudinal chain cancel out [26]. In the present case, the epoxy-amine system under study is a fully cured system. This implies that some of the following characteristics exist in the polymer composite: (a) very high viscosity (b) decrease of dipole moment per unit volume due to the increase of chain length (c) reduction in the number of dipolar groups (d) presence of unreacted epoxy groups and (e) appearance of new dipolar groups like the hydroxyl $(\mathrm{OH})$ groups and tertiary amine groups having specific dipole moments, due to the cross-linking reaction between the epoxy resin and the amine hardener [26].

\subsection{DIELECTRIC PERMITTIVITY}

The variations of dielectric permittivity with frequency for the epoxy nanocomposites having $\mathrm{Al}_{2} \mathrm{O}_{3}$ and $\mathrm{TiO}_{2}$ nano-fillers at different filler concentrations are shown in Figures 3 and 4 respectively. The frequencies of permittivity measurement are in the range of $10^{6}-10^{9} \mathrm{~Hz}$ and when referred to Figure 2, the characteristics will be determined by orientation polarization effects (where dipoles tend to orient themselves to an applied electric field). Since the measurement temperature is maintained constant in this study, its influence on the dielectric permittivity and tan delta behavior can be neglected. The segregation of the different relaxation mechanisms $(\alpha, \beta, \gamma)$ in this study, especially for the chosen experimental frequency range is difficult without studies involving temperature. In the case of epoxy- $\mathrm{TiO}_{2}$ nanocomposites, the formation of ether groups during the curing process due to the reaction of surface hydroxyl groups on the $\mathrm{TiO}_{2}$ surfaces and epoxide rings has been reported [31]. Apart from this, there is a possibility for unreacted dipolar groups to exist which can influence the permittivity of the nanocomposite 
medium. These functional groups having definite dipole moments will contribute to the polarization mechanisms in the epoxy nanocomposites which in turn influences the permittivity of the system. The relaxation time scales for the dipolar groups contributing to each of the relaxation mechanisms separately would be all in the same range and hence it is difficult to isolate the different relaxation behaviors uniquely in this case. The number of such dipolar groups in a cured epoxy system will be significantly less as compared to an uncured epoxy system, so the permittivity values in polymers are usually small and the changes due to the effects of frequency in the current measurement range will also be marginal.

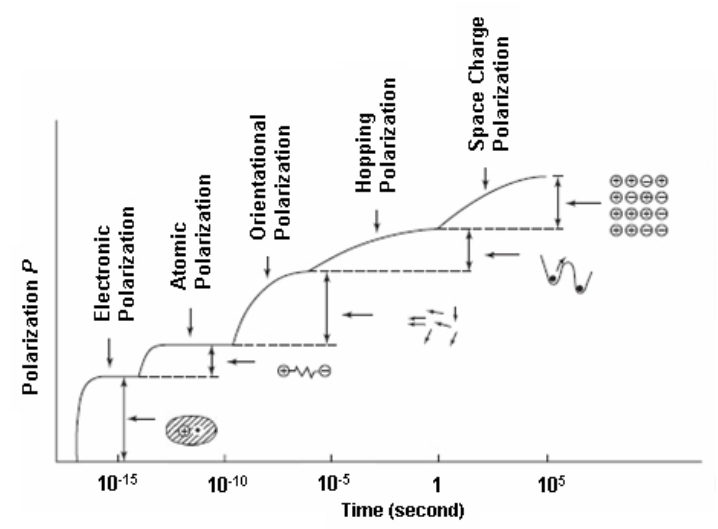

Figure 2. Polarization index in an ideal Debye dielectric (Source: Dielectric Phenomena in Solids by Kwan Chi Kao).

\subsubsection{FREQUENCY DEPENDENCE}

There is only a marginal decrease in the nanocomposite effective permittivity with increasing frequency over the measured frequency range irrespective of the type of filler and filler concentration. The effective permittivity in epoxy nanocomposites depends on the individual permittivities of epoxy and the fillers as well as the filler loadings. This dependence is also known from the effective medium theories and mixing rules used to calculate the effective permittivity in polymer-particle heterogeneous systems [17]. The permittivity of pure epoxy will marginally decrease with increasing frequency in the present frequency range of $1 \mathrm{MHz}-1 \mathrm{GHz}$ due to reduction in the polarizations caused by dipolar groups [17]. Similarly, the permittivity in $\mathrm{TiO}_{2}$ and $\mathrm{Al}_{2} \mathrm{O}_{3}$ also reduces with increasing frequency [32-34]. Therefore, for a combined system of epoxy with these nano-fillers, the overall nanocomposite effective permittivity will slowly reduce with increasing frequency for the frequency range studied.

\subsubsection{LOWER EFFECTIVE PERMITTIVITY}

The other interesting observation evident from the figures is that up to a certain nano-filler concentration, the permittivities of the epoxy nanocomposites are less than that of the unfilled epoxy in the entire frequency range. Figure 3 for $\mathrm{Al}_{2} \mathrm{O}_{3}$-epoxy nanocomposite shows that the values of effective permittivity with $0.1 \%, 0.5 \%$ and $1 \%$ filler concentrations are less than that of the permittivity value in unfilled epoxy. Only with $5 \%$

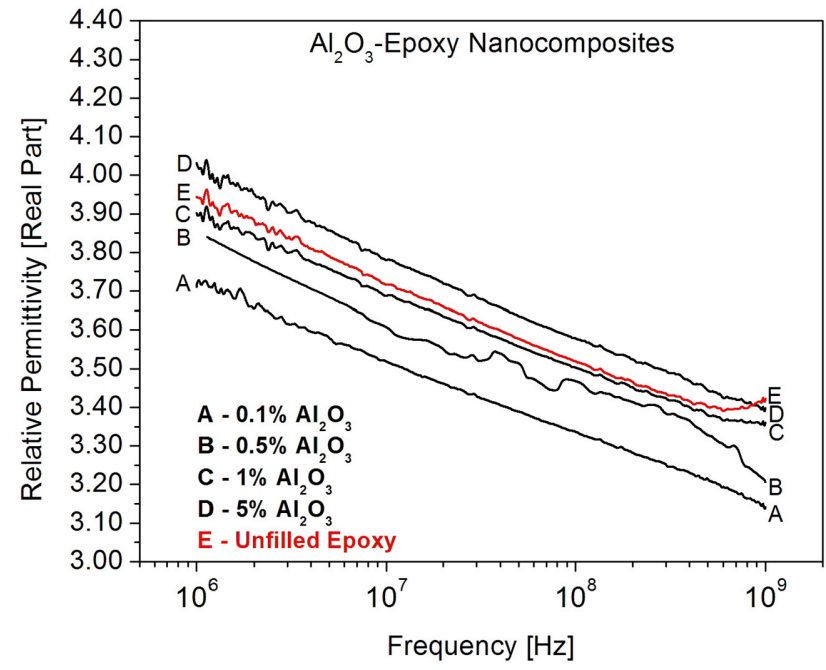

Figure 3. Variations of relative permittivity (real) with respect to frequency in $\mathrm{Al}_{2} \mathrm{O}_{3}$-Epoxy Nanocomposites.

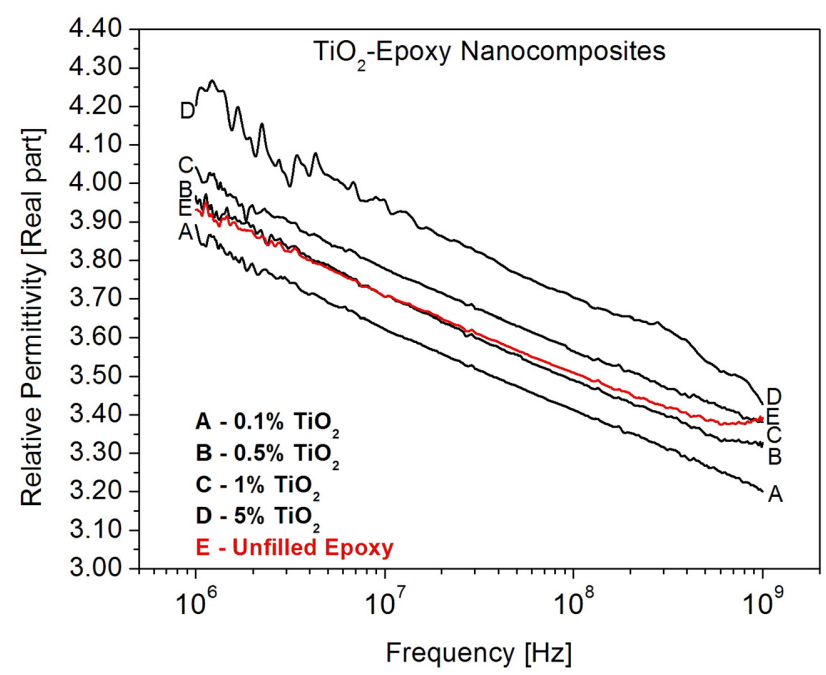

Figure 4. Variations of relative permittivity (real) with respect to frequency in $\mathrm{TiO}_{2}$-Epoxy Nanocomposites.

$\mathrm{Al}_{2} \mathrm{O}_{3}$ concentration, the effective permittivity value is higher than that of the unfilled epoxy value. On the other hand, it can be seen from Figure 4 that over the entire frequency range, only the effective permittivity in $0.1 \%$ nano- $\mathrm{TiO}_{2}$ filled epoxy is found to be lower than the values of unfilled epoxy. With $0.5 \%$ nano- $\mathrm{TiO}_{2}$ concentration, the permittivity values are found to be almost the same as that of unfilled epoxy whereas the nanocomposite effective permittivity is higher than that of unfilled epoxy with $1 \%$ and $5 \%$ nano- $\mathrm{TiO}_{2}$ loadings. A similar observation of a lower effective permittivity in polymer nanocomposites has also been reported elsewhere [35, 36]. But their experimental parameters are different from the present study. Nelson et al [35] reported the results of epoxy nanocomposites with $\mathrm{TiO}_{2}$ fillers of APS (Average Particle Size) $38 \mathrm{~nm}$ at $10 \%$ filler loading as compared to an APS of 50 $\mathrm{nm}$ in the present study. In the other study by Imai et al [36], investigations were performed in epoxy filled with organically modified layered silicates. The current observations in the effective permittivities of epoxy nanocomposites filled with 
nanometer sized $\mathrm{Al}_{2} \mathrm{O}_{3}$ and $\mathrm{TiO}_{2}$ particles are unique since they do not seem to follow the trends as known for polymer microcomposites. Such a reduction in the effective permittivity for few nanofiller concentrations in the epoxy nanocomposites (with different fillers) is only possible if the polarization processes in the nanocomposites are curtailed.

The effective permittivity in nanocomposites is determined by dielectric polarization mechanisms in the bulk of the material. In the present case, they are polarizations associated with epoxy and $\mathrm{TiO}_{2} / \mathrm{Al}_{2} \mathrm{O}_{3}$ and interfacial polarizations at the epoxy-nanoparticle interfaces. It is well known that apart from the nanoparticles, nanocomposites have a large volume fraction of interfaces where interfacial polarizations are most likely to occur. The present investigations utilize uncoated nanoparticles for the experiments which further enhances the prospects of interfacial polarization. But in the range of high frequencies used in the present experiments, interfacial polarizations are unlikely to occur in the nanocomposites. Interfacial or space charge polarizations occur due to the accumulation of space charges at interface boundaries. When an electric field of high frequency as in the present case is applied, the probabilities of these space charges to drift and accumulate at polymer-nanoparticle interfaces become highly remote. Apart from this, most of these space charges usually have large time lags for mobility which makes it even more difficult for them to drift at such high frequencies. Usually, occurrences of interfacial polarizations are observed at lower frequencies of dielectric measurement. Since interfacial phenomenon is an additional polarization mechanism apart from ionic, electronic and dipolar mechanisms, their occurrence in a system is usually associated with distinct variations in the trends (a steep rise) of tan delta and effective permittivity with respect to frequency, especially at high filler concentration. In the present study, such trends in the variations of the dielectric properties are not seen. Further, available results of space-charge measurements using Pulsed Electro-acoustic technique (PEA) in nanocomposites indicate that space-charges in the bulk of these materials are mitigated $[7,11]$. It is a fact that the individual permittivities of $\mathrm{Al}_{2} \mathrm{O}_{3}$ and $\mathrm{TiO}_{2}$ are higher than that of pure epoxy and their polarization mechanisms are intrinsic. This intrinsic nature of the polarization in nanoparticles cannot therefore contribute to a lowering of the effective permittivity in the nanocomposite. Thus, there remain only the individual polarization processes in epoxy that can lower the effective permittivity in epoxy nanocomposites. Naturally, based on the results, we would expect the polarization associated with epoxy to decrease.

As mentioned earlier, the polarization mechanism in epoxy at the frequencies used in the current study is due to the orientation of dipolar groups with respect to the electric field. This orientational polarization process in epoxy can be reduced only if the movements of the contributing dipolar groups are constrained. As a matter of fact, occurrences of reduced polymer chain mobility in polymer nanocomposites have already been reported. Research on the mechanical behaviors in polymer nanocomposites has shown significant improvements in several properties [24]. Limited laboratory experiments [37, 38] as well as molecular dynamics simulations $[39,40]$ have conjectured that these enhancements in the mechanical characteristics are due to restrictions in the mobility of polymer chains in the nanocomposite caused by the strong interactions of the polymer chains with the highly charged nano-fillers. Depending on the interaction mechanism of the polymer with the nanoparticle, an interfacial polymer nanolayer is reported to form on the nanoparticle surface which is highly immobile due to the strong bonding of the polymer chains and the particle surface [37]. When these immobile nanolayer formations are extended to all the nanoparticles in a polymer matrix, it can be expected that the mobility of the polymer segments or chains interacting with these nanoparticles in the nanocomposite are restricted. It has also been mentioned in some other studies that when the length scales of the polymer chain and the filler particles come closer, the interface wall-wall distances between particles become smaller and a secondary polymer chain network forms which causes entanglements [41, 42]. These entanglements further reduce the mobility of polymer chains in the nanocomposite. The immobility and entanglement dynamics of the polymer chains are a function of the filler concentration and only those polymer chains which come in contact with the nanoparticles will become immobile or entangled. The same theories as above can be extended to the case of epoxy nanocomposites in the present study too. In all probability, the epoxy polymer segments interact with the $\mathrm{Al}_{2} \mathrm{O}_{3}$ and $\mathrm{TiO}_{2}$ nanofillers causing a restriction in the mobility of these polymer segments. This restriction in turn influences the occurrence of a lower effective permittivity in nanocomposites (as seen for few filler fractions) with both the fillers.

The interaction mechanisms at the interfacial area and the consequent nanolayer formation in polymer nanocomposites are reported to influence their glass transition temperatures $\left(T_{g}\right)$. An increase in $T_{g}[37,43]$ has been observed in few cases whereas few other reports cite a reduction in $T_{g}$ when nanofillers are added to polymers [44], but most of the proposed theories are still inconclusive. To have a better understanding of the interfacial interaction in the current study, the glass transition temperatures of the epoxy nanocomposites with both $\mathrm{Al}_{2} \mathrm{O}_{3}$ and $\mathrm{TiO}_{2}$ fillers were also measured. Representative DSC curves showing the $\mathrm{T}_{\mathrm{g}}$ values are shown in Figure 5 whereas the variations in the $T_{g}$ values for all the epoxy nanocomposite compositions used in the current investigations are shown in Figure 6. It can be ascertained from Figure 6 that for all the filler loadings with both the fillers, the glass transition temperatures in all the nanocomposites are less than that of unfilled epoxy. A decrease in $T_{g}$ in polymer composites can also happen because of changes induced during processing like molecular weight, tacticity and cross-linking density. But, in the present investigations, the processing method utilized to prepare the epoxy nanocomposites is the same for the different fillers as well as filler concentrations. Therefore, it is expected that the changes in $T_{g}$ are due to the effect of nanoparticles only. The $\mathrm{T}_{\mathrm{g}}$ reduction in nanocomposites raises another question - if the polymer chain mobility is restricted in nanocomposites, the $T_{g}$ values should increase rather than decrease as in the present case. Few studies in polymer nanocomposites have suggested 


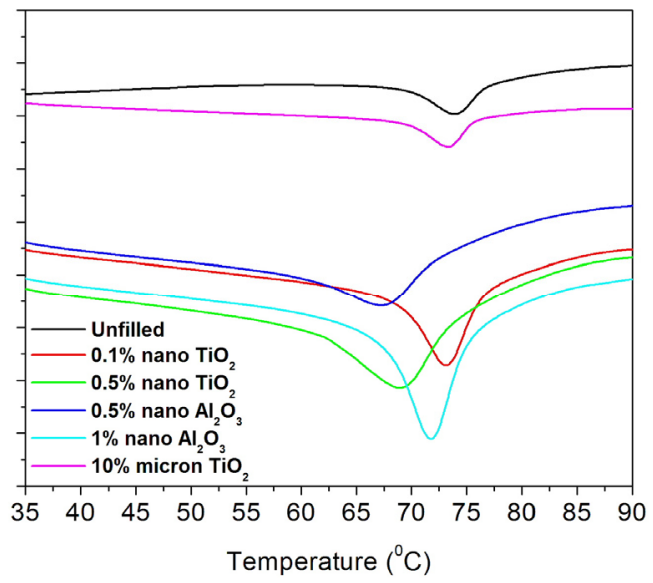

Figure 5. Representative DSC curves for the epoxy nanocomposites.

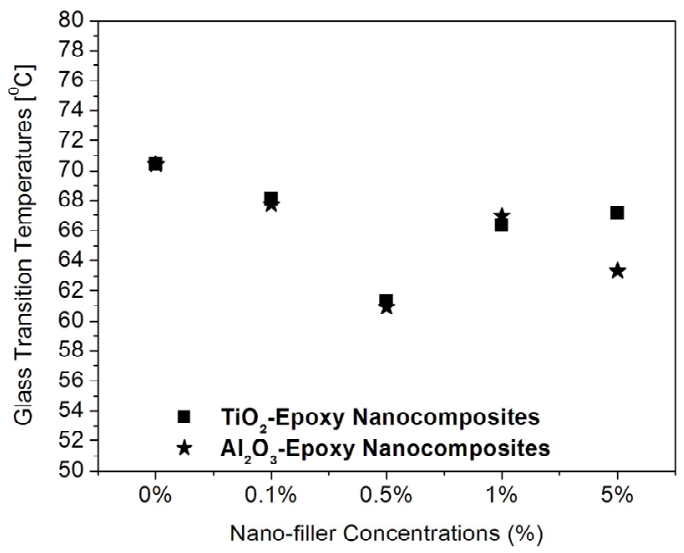

Figure 6. Variations of $\mathrm{T}_{\mathrm{g}}$ in epoxy nanocomposites.

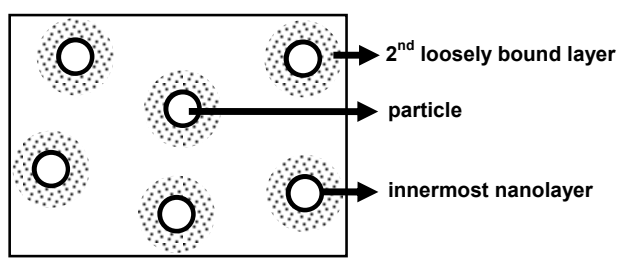

Figure 7. Indicative picture of the interface in the nanocomposite (Dual layer model) [37].

that polymer-nanoparticle interactions actually lead to the formation of more than one nanolayer around the nanoparticle $[37,43]$. In addition to the formation of the immobile polymer nanolayer close to the particle, another polymer layer with a thickness slightly more than that of the immobile layer forms over this. An indicative picture is shown in Figure 7. The polymer segments in this extended layer is reported to be loosely bound and they relax faster causing a reduction in the nanocomposite glass transition temperature as seen in the current study [37]. At low nano-filler concentrations, the immobile nanolayers closest to the particle surface would be very thin which in turn allows the nanoparticles to have a far stronger interaction with the second layer of loosely bound polymer [37]. These interfacial phenomena are dependent on filler dispersion in the nanocomposite and since synthesizing a totally agglomeration free nanocomposite is difficult; the phenomena occurring at agglomerated or clustered nano-filler locations are difficult to comprehend at this point. Usually at lower filler concentrations, the filler distributions in the polymer are uniform with large inter-particle distances and so the interfacial effects in nanocomposites may be much more pronounced. Based on the discussions above, there seems to be a strong correlation between the mentioned theories and the effective permittivity results obtained in the present study. To a certain extent, the theories are able to explain the reduction in the nanocomposite effective permittivity and further discussions are beyond the scope of this paper.

\subsubsection{EFFECT OF NANOPARTICLE LOADING}

It can be seen from Figures 3 and 4 that with increasing nanofiller concentrations in epoxy, there is an increase in the nanocomposite effective permittivity with both $\mathrm{TiO}_{2}$ and $\mathrm{Al}_{2} \mathrm{O}_{3}$ fillers. The effective permittivity is a function of the epoxy permittivity, filler permittivity and the concentration of fillers in the composite. The permittivity associated with the epoxy component in the nanocomposite will still exist in spite of the polymer chain immobilizations. This is because the nanocomposite will have several dipolar groups which are not interacting with the nanoparticles and so they would be free to orient with the applied electric field. Assuming the case of micrometer sized $\mathrm{TiO}_{2}$ (permittivity $\approx 100$ ) and $\mathrm{Al}_{2} \mathrm{O}_{3}$ (permittivity $\approx 9$ ) filled epoxy and using Lichteneker-Rother mixing rule [equation (1)], it is found that the addition of $0.1 \%$ $\mathrm{TiO}_{2}$ in epoxy results in a variation of the effective permittivity by $0.24 \%$ whereas with $\mathrm{Al}_{2} \mathrm{O}_{3}$ filler, the variation is just $0.07 \%$.

$$
\log \varepsilon_{\mathrm{c}}=\mathrm{x} \log \varepsilon_{1}+\mathrm{y} \log \varepsilon_{2}
$$

where, $\varepsilon_{\mathrm{c}}$ is the effective composite permittivity, $\varepsilon_{1}$ and $\varepsilon_{2}$ are the permittivity of filler and polymer and $\mathrm{x}, \mathrm{y}$ are the concentrations of filler and polymer respectively.

Although Lichteneker-Rother mixing rule cannot be directly used in nanocomposites, the trends would be very similar. Therefore, for $0.1 \%$ nano-filler concentration in epoxy with both the fillers, it is expected that the contributions of the filler permittivity to the effective nanocomposite permittivity would be very minimal implying that the effective permittivity at this loading will be mainly governed by the epoxy contributions. Additionally, it was also mentioned in the earlier section that at low filler loadings, the interaction of the nanoparticle with the loose polymer would be very strong. Thus, at $0.1 \%$ nano-filler loading, the nanocomposite effective permittivity will be governed by the occurrence of two processes - minimum effect of nano-filler permittivity and strong polymer-nanoparticle interactions. This is probably the reason why the lowest effective permittivities in both $\mathrm{TiO}_{2}$ and $\mathrm{Al}_{2} \mathrm{O}_{3}$ filled epoxy nanocomposites are observed at a filler loading of $0.1 \%$. For polymer microcomposites, Lichteneker's rule predicts an increase in the composite permittivity with addition of micrometer sized fillers. Similarly, excluding the unfilled epoxy permittivity trend from the Figures 3 and 4 for a moment, it can be seen that the addition of $\mathrm{TiO}_{2}$ and $\mathrm{Al}_{2} \mathrm{O}_{3}$ nanoparticles to epoxy beyond $0.1 \%$ increases the effective permittivity in the nanocomposites. The highest effective permittivity in the nanocomposites with both the fillers is observed at the highest filler concentration of 5\%. An increase in the nano-filler 
concentration in epoxy results in an increase in the number of nanoparticles and with the individual permittivities of each of these particles contributing to the permittivity of the composite, there is an increase in the effective permittivity. As discussed earlier, the lowering of effective permittivity is assumed to be due to immobilization of polymer chains and in turn the extent of immobility is a function of the filler concentration. The more the fillers, the more immobile are the polymer chains. So indirectly the effective permittivity should reduce with increasing nano-filler concentration. But again, the effective permittivity is also a function of the number of nanofillers in the matrix and with increasing filler concentration, the permittivity will tend to go up. This interaction dynamics between these two processes which happen simultaneously in the nanocomposite is a complex one to understand and needs further study. But, in all probability, the rate of chain immobilization and the rate of permittivity enhancement with respect to the filler loading, will determine which way the nanocomposite effective permittivity will change. This is where the permittivity of the filler will have an effect since the permittivity enhancement will depend on this value too. This permittivity effect in the current results is discussed in the next section. Another important factor to be considered here is that for a fixed filler loading in the nanocomposite, the filler density will also determine the number of nanoparticles in the material bulk. This is apart from the knowledge that when compared to the number of fillers in polymer microcomposites, the number of fillers in polymer nanocomposites is more for the same filler concentration. Since the number of particles have an important bearing on the effective permittivity of nanocomposites, straightforward comparisons of the effective permittivity variations in two different epoxy nanocomposites with different types of fillers pose an even bigger challenge.

\subsubsection{EFFECT OF FILLER PERMITTIVITY}

As mentioned, two different fillers $\left(\mathrm{TiO}_{2}\right.$ and $\left.\mathrm{Al}_{2} \mathrm{O}_{3}\right)$ with a wide difference in their permittivities are used in the current experiments. An inspection of the effective permittivities of the epoxy nanocomposites in Figures 3 and 4 shows that the filler type has a significant effect. In $\mathrm{Al}_{2} \mathrm{O}_{3}$-epoxy nanocomposites, a lower effective permittivity is observed up to a nano-filler concentration of $1 \%$ whereas in $\mathrm{TiO}_{2}$ filled systems, the same effect is seen only at $0.1 \%$ filler loading. This observation is most likely due to the differences in permittivities between $\mathrm{TiO}_{2}$ and $\mathrm{Al}_{2} \mathrm{O}_{3}$. Due to the lower permittivity value of $\mathrm{Al}_{2} \mathrm{O}_{3}(\approx 9)$ as compared to $\mathrm{TiO}_{2}(\approx 100)$, the influence of increasing $\mathrm{Al}_{2} \mathrm{O}_{3}$ nanofillers on the effective permittivity of the composite would be less when compared to the same effect caused by the addition of $\mathrm{TiO}_{2}$ nanofillers. Considering the example of a polymer microcomposite again as earlier and using equation (1), calculations show that with $\mathrm{TiO}_{2}$ fillers, there is a variation of around $15 \%$ in the effective permittivities between $0.1 \%$ and $5 \%$ filler loading. But in the case of $\mathrm{Al}_{2} \mathrm{O}_{3}$ as filler, this same difference is only around $3 \%$, which is mainly due to its low permittivity value. Although in nanocomposites, the number of particles will be

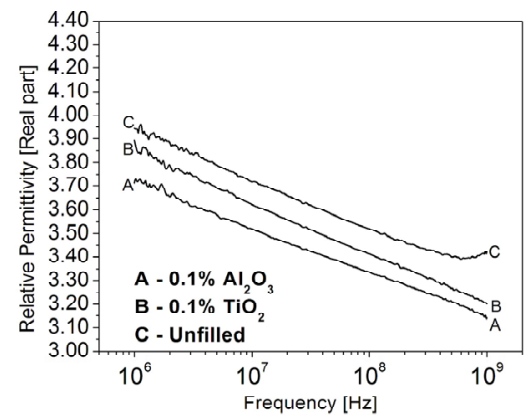

(a) $0.1 \%$ filler loading

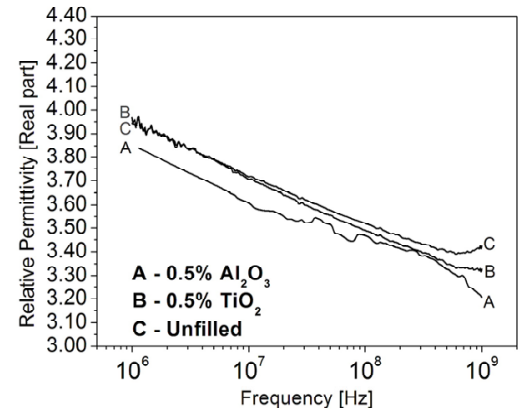

(b) $0.5 \%$ filler loading

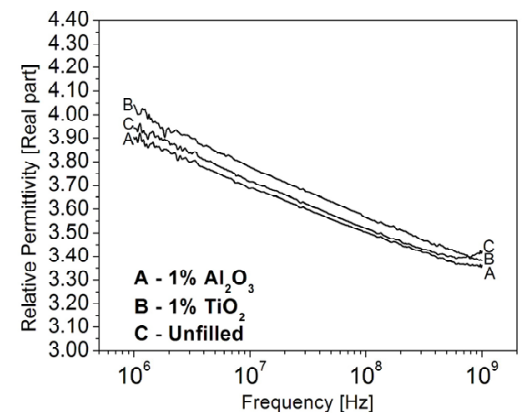

(c) $1 \%$ filler loading

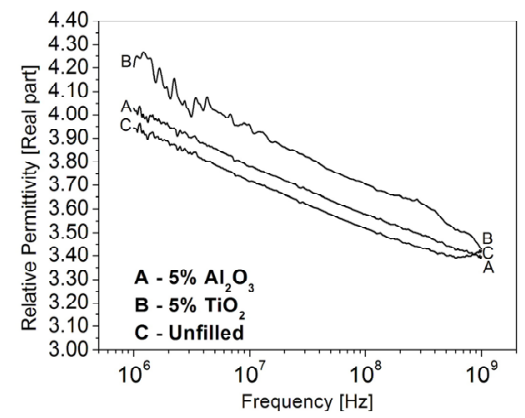

(d) $5 \%$ filler loading

Figure 8. Comparisons of effective permittivity between $\mathrm{Al}_{2} \mathrm{O}_{3}$ and $\mathrm{TiO}_{2}$ filled epoxy nanocomposites at different filler loadings.

different than the number of particles in microcomposites, addition of $\mathrm{Al}_{2} \mathrm{O}_{3}$ nanoparticles beyond $0.1 \%$ and upto $5 \%$ in epoxy won't lead to a significant increase in the effective nanocomposite permittivity as compared to the case of $\mathrm{TiO}_{2}$ nano-filler addition. This effect confirms that depending on the permittivity of the nano-filler, there is a minimum filler concentration level, up to which, the value of nanocomposite effective permittivity will be less than the unfilled epoxy value. The result is significant because, to achieve a material with a 
lower effective permittivity for use in the $1 \mathrm{MHz}-1 \mathrm{GHz}$ frequency range, an epoxy nanocomposite can be designed with a nano-filler material of very low dielectric constant.

A comparison of the effective permittivities between $\mathrm{Al}_{2} \mathrm{O}_{3}$ and $\mathrm{TiO}_{2}$ filled epoxy nanocomposites for different filler weight fractions in the polymer matrix are made and they are shown in Figure 8. It can be seen from the figure that for a fixed filler concentration at a particular frequency, the effective nanocomposite permittivities with $\mathrm{TiO}_{2}$ are always higher than the values obtained with $\mathrm{Al}_{2} \mathrm{O}_{3}$ as the filler. This observation can be due to a combination of two different processes, one caused by the nano-filler permittivity and the other by the nano-filler concentration. The effect of filler permittivity is already discussed above and since $\mathrm{TiO}_{2}$ has a permittivity value higher than $\mathrm{Al}_{2} \mathrm{O}_{3}$, the effective nanocomposite permittivity with $\mathrm{TiO}_{2}$ is always more than when $\mathrm{Al}_{2} \mathrm{O}_{3}$ is the filler. In another aspect, since the density of $\mathrm{Al}_{2} \mathrm{O}_{3}$ (density $\approx 3.7$ ) is marginally less than that of $\mathrm{TiO}_{2}$ (density $\approx 3.9$ ), for the same filler loading, the epoxy nanocomposite with $\mathrm{Al}_{2} \mathrm{O}_{3}$ as the filler will have more number of $\mathrm{Al}_{2} \mathrm{O}_{3}$ nanoparticles as compared to the case when $\mathrm{TiO}_{2}$ is the filler. Especially with fillers in nanometer scales, this difference in the number of particles can be very significant. Thus, for a fixed filler loading, $\mathrm{Al}_{2} \mathrm{O}_{3}$ nanofillers in the epoxy nanocomposite will introduce more interfaces causing additional restrictions to the polymer chain mobility when compared to $\mathrm{TiO}_{2}$ nanofillers. This enhanced chain mobility restrictions with $\mathrm{Al}_{2} \mathrm{O}_{3}$ nanofillers coupled with the effect of a lower $\mathrm{Al}_{2} \mathrm{O}_{3}$ permittivity, will force the effective permittivity of $\mathrm{Al}_{2} \mathrm{O}_{3}$-epoxy nanocomposites to values lower than that of the $\mathrm{TiO}_{2}$ filled ones. Although both these processes will be active in the epoxy nanocomposites, it is difficult to segregate their individual contributions.

\subsection{TAN DELTA}

Tan delta variations with respect to frequency in epoxy nanocomposites with $\mathrm{Al}_{2} \mathrm{O}_{3}$ and $\mathrm{TiO}_{2}$ nanofillers are shown in Figures 9 and 10 respectively. One can see from the graphs that fluctuations exist in the tan delta data (usually occurs during high frequency measurements) of nanocomposites with both $\mathrm{TiO}_{2}$ and $\mathrm{Al}_{2} \mathrm{O}_{3}$ fillers. It would be appropriate to mention here that these fluctuations in the tan delta values should not be mistaken for loss peaks. In polymers or their composites, loss tangent is a function of the electrical conductivity (which depends on the charge carrier mobility) and the applied excitation frequency. It can be seen from these graphs that in both the $\mathrm{Al}_{2} \mathrm{O}_{3}$ and $\mathrm{TiO}_{2}$ filled epoxy nanocomposites, there is a marginal (but continuous) decrease in $\tan$ delta values with increasing frequency for all filler concentrations. The most likely reason for this observation is attributed to a decrease in electrical conductivity in the epoxy nanocomposites with increasing frequency which is caused by the inability of the charge carriers to traverse the thickness of the material at the higher frequencies. Figure 9 shows that in $\mathrm{Al}_{2} \mathrm{O}_{3}$ filled epoxy nanocomposites, at lower frequency ranges $(1-5 \mathrm{MHz})$, the incorporation of fillers do not introduce too much variations in the tan delta values with respect to the

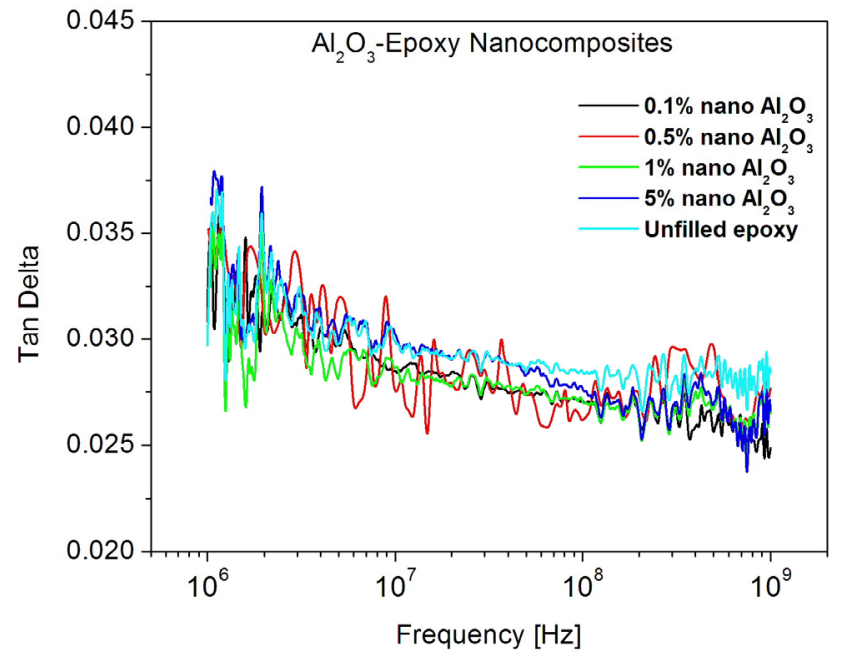

Figure 9. Variations of tan delta with respect to frequency in $\mathrm{Al}_{2} \mathrm{O}_{3}$-Epoxy Nanocomposites.

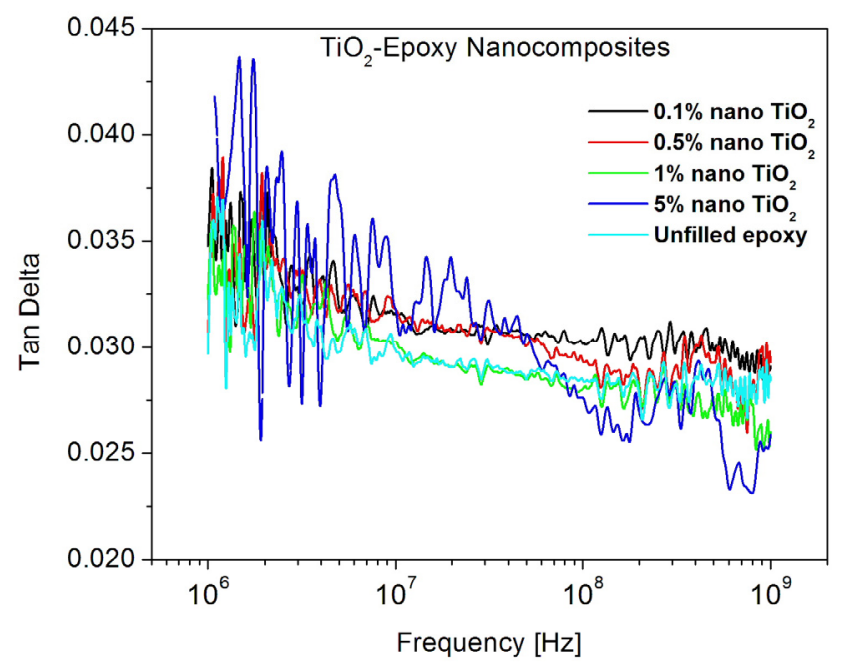

Figure 10. Variations of tan delta with respect to frequency in $\mathrm{TiO}_{2}$ Epoxy Nanocomposites.

unfilled epoxy value. Although the incorporation of inorganic nanofillers into the epoxy matrix will most likely introduce more sources of charge carriers, their effect on the tan delta values are not observed. As the frequency increases, the tan delta values in filled epoxy for all filler concentrations are observed to reduce in comparison to the unfilled epoxy tan delta. But tan delta variations between nanocomposites with different filler loadings are marginal. The occurrence of a lower tan delta value in nanocomposites can be due to a lower electrical conductivity in them at those frequencies. At high frequencies, the motion of charge carriers contributing to the conductivity primarily occur along polymer chains [45]. A barrier to the charge transport in polymers (causing reduction in electrical conductivity) can occur due to defects, inter-chain charge transport and transport through interfaces. Probably, in nanocomposites, the presence of a large number of interfaces and polymer chain entanglements inhibit the motion of charges in the system, which in turn causes a reduction in the electrical conductivity (hence a lower tan delta value). 
Unlike above, a slightly different observation could be made in the case of $\mathrm{TiO}_{2}$ nanocomposites from Figure 10. At lower frequencies, the tan delta values in the filler loaded epoxy are marginally higher than the value of unfilled epoxy, but as the frequency increases, only tan delta values with $0.1 \%$ and $0.5 \% \mathrm{TiO}_{2}$ in the nanocomposite are higher. At higher frequencies, tan delta with $1 \%$ and $5 \%$ filler loading in epoxy reduce to a value lower than that of unfilled epoxy. This observation is slightly difficult to explain with the available results and understanding. There are basically two different interacting processes which might influence tan delta behavior in nanocomposites. The first one is the number of charge carriers available for electrical conduction and the other is the number of interfaces and polymer chain entanglements in the bulk. $\mathrm{TiO}_{2}$ has a higher dielectric permittivity, so the number of charge carriers introduced into the nanocomposite would be higher in $\mathrm{TiO}_{2}$ filled epoxy. Therefore, the interplay of the number of charge carriers, the interface barriers and chain entanglements towards the electrical conductivity in the $\mathrm{TiO}_{2}-$ epoxy nanocomposites at these frequencies are complicated and they are currently under investigation.

Overall, the introduction of nano-fillers into epoxy does not seem to significantly alter the tan delta values in epoxy nanocomposites, especially for the examined filler concentrations and frequencies. This is true with different types of fillers too since with both $\mathrm{TiO}_{2}$ and $\mathrm{Al}_{2} \mathrm{O}_{3}$ nanofillers, tan delta is in the range of $0.025-0.0375$. These nanocomposite observations are different from the known tan delta behaviors in microcomposites where tan delta is usually seen to increase significantly with increasing filler concentrations $[13,15]$.

\section{CONCLUSIONS}

The characteristics of dielectric permittivity and tan delta in epoxy nanocomposites with $\mathrm{Al}_{2} \mathrm{O}_{3}$ and $\mathrm{TiO}_{2}$ nano-fillers over a frequency range of $1 \mathrm{MHz}-1 \mathrm{GHz}$ is found to be different from the expected trends seen in polymer microcomposites. The incorporation of nano-fillers to an epoxy matrix reduces the permittivity of the nanocomposites for specific filler percentages when compared to that of unfilled epoxy and such phenomena was never observed in epoxy microcomposites earlier. But as expected, the effective permittivity increases with increasing filler concentration. The lowering of the nanocomposite effective permittivity has been found to be a function of the filler concentration and the filler permittivity. It is suggested that this unique permittivity behavior is due to the restrictions imposed on the mobility of dipolar groups in the nanocomposite by the interaction of the nanoparticle and the polymer. To further understand this behavior, the glass transition temperatures of the nanocomposites were also measured. A dual layer model used to explain mechanical behaviors in polymer nanocomposites seems to fit the dielectric permittivity characteristics observed in the current study. In the case of tan delta, for both the types of nanocomposites, changes in the filler concentration levels do not seem to impart significant variations in the values at all the frequencies. For $\mathrm{Al}_{2} \mathrm{O}_{3}$-epoxy nanocomposites, tan delta at all the filler concentrations were less than or equal to the unfilled epoxy value over the frequency range studied. But in $\mathrm{TiO}_{2}$ epoxy nanocomposites, although the tan delta values do not deviate much with respect to the value for unfilled epoxy, there are variations with respect to the frequency. In summary, it is the epoxy-filler interface which determines the behaviors of the effective permittivity and tan delta values in epoxy nanocomposites and a complete understanding of the interfacial phenomena is of utmost importance to fully understand not only these two dielectric parameters, but also several other important electrical properties.

\section{ACKNOWLEDGMENT}

The authors would like to thank the Nanotechnology Centre and the Materials Engineering department of the Indian Institute of Science, Bangalore for the access to the FESEM facility and the DSC equipment respectively. We are also grateful to Mr. Prasad and Mr. Padaikathan for their help in the DSC experiments. Further, the first author is grateful to the IEEE DEIS for the award of the Graduate Fellowship which enabled him to buy the $\mathrm{Al}_{2} \mathrm{O}_{3}$ nanofillers for this study.

\section{REFERENCES}

[1] F. Carmona, "Conducting Filled Polymers", Physica A, Vol. 157, pp. 461-469, 1989.

[2] Y. Bai, Z. -Y. Cheng, V. Bharti, H. S. Xu and Q. M. Zhang, "High dielectric-constant ceramic-powder polymer composites", Appl. Phys. Letters, Vol. 76, No. 25, pp. 3804-3806, 2000.

[3] M. M. Ueki and M. Zanin, "Influence of additives on the dielectric strength of High-density Polyethylene", IEEE Trans. Dielectr. Electr. Insul., Vol. 6, No. 6, pp. 876-881, 1999.

[4] P. B. Messersmith and E. P. Giannelis, "Synthesis and Characterization of Layered Silicate-Epoxy Nanocomposites", Chem. Mater., Vol. 6, pp. 1719-1725, 1994.

[5] S. S. Ray and M. Okamoto, "Polymer/layered silicate nanocomposites: a review from preparation to processing", Prog. Polym. Sci., Vol. 28, pp. 1539-1641, 2003.

[6] R. Gensler, P. Groppel, V. Muhrer and N. Muller, "Applications of Nanoparticles in Polymers for Electronic and Electrical Engineering", Particle and Particle Systems Characterization, Vol. 19, pp. 293-299, 2002.

[7] T. Tanaka, "Dielectric Nanocomposites with Insulating Properties", IEEE Trans. Dielectr. Electr. Insul., Vol. 12, No. 5, pp. 914-928, 2005.

[8] Y. Cao, P. C. Irwin and K. Younsi, "The Future of Nanodielectrics in the Electrical Power Industry”, IEEE Trans. Dielectr. Electr. Insul., Vol. 11, pp. 797-807, 2004.

[9] T. Imai, F. Sawa, T. Ozaki, Y. Inoue, T. Shimizu and T. Tanaka, "Comparison of Insulation Breakdown Properties of Epoxy Nanocomposites under Homogeneous and Divergent Electric Fields", IEEE Conf. Electr. Insul. Dielectr. Phenomena (CEIDP), pp. 306-309, 2006.

[10] T. J. Lewis, "Interfaces are the Dominant Feature of Dielectrics at the Nanometric Level", IEEE Trans. Dielectr. Electr. Insul., Vol. 11, pp. 739-753, 2004.

[11] M. Roy, J. K. Nelson, R. K. MacCrone and L. S. Schadler, "Polymer Nanocomposite Dielectrics - The Role of the Interface", IEEE Trans. Dielectr. Electr. Insul., Vol. 12, pp. 629-643, 2005.

[12] P. M. Ajayan, L. S. Schadler and P. V Braun, Nanocomposite Science and Technology, Chapter 2, Wiley 2003.

[13] L. Ramajo, M. Reboredo and M. Castro, "Dielectric response and relaxation phenomena in composites of epoxy resin with $\mathrm{BaTiO}_{3}$ particles", Composites: Part A, Vol. 36, pp. 1267-1274, 2005.

[14] S. Yu, P. Hing and X. Hu, "Dielectric Properties of PolystyreneAluminum-Nitride Composites", J. Appl. Phys., Vol. 88, pp. 398-404, 2000. 
[15] M. J. Yim, W. Kwon and K. W. Paik, "Effect of filler content on the dielectric properties of anisotropic conductive adhesives materials for high-frequency flip-chip interconnection", Materials Sci. Eng. B, Vol. 126, pp. 59-65, 2006.

[16] J W Liou and B S Chiou, "Dielectric tunability of barium strontium titanate/silicone rubber composite", J. Phys.: Condensed Matter, Vol. 10, pp. 2773-2786, 1998

[17] C. Brosseau, P. Queffelec and P. Talbot, "Microwave characterization of filled polymers", J. Appl. Phys., Vol. 89, pp. 4532-4540, 2001.

[18] J. C. Fothergill, J. K. Nelson and M. Fu, "Dielectric Properties of Epoxy Nanocomposites containing $\mathrm{TiO}_{2}, \mathrm{Al}_{2} \mathrm{O}_{3}$ and $\mathrm{ZnO}$ fillers", IEEE Conf. Electr. Insul. Dielectr. Phenomena (CEIDP), pp. 406-409, 2004

[19] J. I. Hong, P. Winberg, L. S. Schadler, R. W. Siegel, "Dielectric Properties of zinc oxide/polyethylene nanocomposites", Materials Letters, Vol. 59, pp. 473-476, 2005.

[20] D. Fragiadakis, E. Logakis, P. Pissis, V. Yu. Kramarenko, T. A. Shantalii, I. L. Karpova, K. S. Dragan, E. G. Privalko, A. A. Usenko and V. P. Privalko, "Polyimide/silica nanocomposites with low values of dielectric permittivity", J. Phys.: Conf. Series 10 (2nd Conf. Microelectronics, Microsystems and Nanotechnology), pp. 139-142, 2005.

[21] H. Windlass, P. M. Raj, D. Balaraman, S. K. Bhattacharya and R. R. Tummala, "Polymer Ceramic Nanocomposite Capacitors for System-OnPackage (SOP) Applications", IEEE Trans. Advanced Packaging, Vol. 26, pp. 10-16, 2003.

[22] Z.-M. Dang, L.-Z. Fan, S.-J. Zhao and C.-W. Nan, "Preparation of nanosized $\mathrm{ZnO}$ and dielectric properties of composites filled with nanosized ZnO”, Materials Sci. Eng., Vol. B99, pp. 386-389, 2003.

[23] J. Wua and L. Kong, "High microwave permittivity of multiwalled carbon nanotube composites", Appl. Phys. Letters, Vol. 84, pp. 49564958, 2004.

[24] J. Jordan, K. I. Jacob, R. Tannenbaum, M. A. Sharaf and I. Jasiuk, "Experimental Trends in Polymer Nanocomposites - A Review", Materials Sci. Eng. A, Vol. 393, No. 1-2, pp. 1-11, 2005.

[25] S. Singha and M. J. Thomas, "Polymer composite/nanocomposite processing and its effect on the electrical properties", IEEE Conf. Electr. Insul. Dielectr. Phenomena (CEIDP), pp. 557-560, 2006.

[26] A. Livi, V. Levita and P. A. Rolla, "Dielectric Behavior at Microwave Frequencies of an epoxy resin during crosslinking", J. Appl. Polymer Sci., Vol. 50, 1583-1590, 1993.

[27] J. P. Eloundou, "Dipolar relaxations in an epoxy-amine system", European Polymer J., Vol. 38, pp. 431-438, 2002.

[28] L. Zong, S. Zhou, R. Sun, L. C. Kempel and M. C. Hawley, "Dielectric Analysis of a Crosslinking Epoxy Resin at a High Microwave Frequency", J. Polymer Sci.: Part B: Polymer Phys., Vol. 42, pp. 28712877,2004

[29] R. Casalini, D. Fioretto, A. Livi, M. Lucchesi and P. A. Rolla, "Influence of the glass transition on the secondary relaxation of an epoxy resin", Phys. Rev. B, Vol. 56, pp. 3016-3021, 1997.

[30] E. Butta, A. Livi, C. Levita and P. A. Rolla, Dielectric Analysis of an Epoxy Resin during Cross-Linking", J. Polymer Sci.: Part B: Polymer Phys., Vol. 33, pp. 2253-2261, 1995.

[31] J. K. Nelson, L. A. Utracki, R. K. Maccrone and C. W. Reed, "Role of the Interface in Determining the Dielectric Properties of Nanocomposites", IEEE Conf. Electr. Insul. Dielectr. Phenomena (CEIDP), pp. 314-317, 2004

[32] H. B. Lal and K. G. Srivastava, "Dielectric properties of normal, reduced and specially reduce rutile $\left(\mathrm{TiO}_{2}\right)$ single crystals at room temperature", Canadian J. Phys., Vol. 47, No. 3, pp. 3-6, 1969.

[33] L. D. Zhang, H. F. Zhang, G. Z. Wang, C. M. Mo and Y. Zhang "Dielectric behavior of nano-TiO ${ }_{2}$ bulks", Phys. Stat. Sol. Vol. 157, pp. 483-491, 1996

[34] J. de los Santos, D. Garcia and J. A. Eiras, "Dielectric characterization of materials at microwave frequency range", Materials Research, Vol. 6, pp. 97-101, 2002.

[35] J. K. Nelson, J. C. Fothergill, L. A. Dissado and W. Peasgood, "Towards an understanding of Nanometric dielectrics", IEEE Conf. Electr. Insul. Dielectr. Phenomena (CEIDP), pp. 295-298, 2002.
[36] T. Imai, Y. Hirano, H. Hirai, S. Kojima and T. Shimizu, "Preparation and Properties of Epoxy-Organically Modified Layered Silicate Nanocomposites", IEEE Intern. Sympos. Electr. Insul. (ISEI), pp. 379383,2002

[37] G. Tsagaropoulos and A. Eisenberg, "Dynamic Mechanical Study of the Factors Affecting the Two Glass Transition Behavior of Filled Polymers. Similarities and Differences with Random Ionomers", Macromolecules, Vol. 28, pp. 6067-6077, 1995.

[38] R. Mansencal, B. Haidar, Alain Vidal, L. Delmotte and J. -M. Chezeau, "High-resolution solid-state NMR investigation of the filler-rubber interaction: 2. High-speed $\left[{ }^{1} \mathrm{H}\right]$ magic-angle spinning NMR spectroscopy in carbon-black-filled polybutadiene", Polymer Intern., Vol. 50, pp. 387394, 2001.

[39] G. J. Papakonstantopoulos, M. Doxastakis, P. F. Nealey, J.-L. Barrat and J. J. de Pablo, "Calculation of local mechanical properties of filled polymers", Phys. Rev. E, Vol. 75, pp. 031803 (1) - 038103 (13), 2007.

[40] R. C. Picu and A. Rakshit, "Dynamics of free chains in polymer nanocomposites", J. Chem. Phys., Vol. 126, pp. 144909 (1) - 144909 (6), 2007.

[41] R. C. Picu and M. S. Ozmusul, "Structure of linear polymeric chains confined between impenetrable spherical walls", J. Chem. Phys., Vol. 118, pp. 11239-11248, 2003

[42] S. S. Sternstein and Ai-Jun Zhu, "Reinforcement mechanism of Nanofilled polymer melts as elucidated by nonlinear viscoelasatic behavior", Macromolecules, Vol. 35, pp. 7262-7273, 2002.

[43] F. W. Starr, T. B. Schrøder and S. C. Glotzer, "Effects of a nanoscopic filler on the structure and dynamics of a simulated polymer melt and the relationship to ultrathin films", Phys. Rev. E, Vol. 64, pp. 021802 (1) $021802(5), 2001$.

[44] B. J. Ash, L. S. Schadler and R. W. Siegel, "Glass transition behavior of Alumina/polymethylmethacrylate nanocomposites", Materials Letters, Vol. 55, pp. 83-87, 2002

[45] P. Prins, F. C. Grozema, J. M. Schins and L. D. A. Siebbeles, "Frequency dependent mobility of charge carriers along polymer chains with finite length”, Phys. Stat. Sol. (b), Vol. 243, pp. 382-386, 2006.

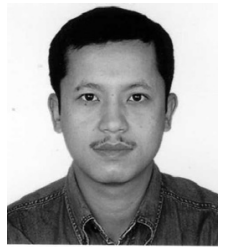

Santanu Singha (S'99) was born in Assam, India in 1975. He received the B.E. degree in electrical engineering from Assam Engineering College, Guwahati, India in 1997 and the M.Sc. (Engg.) degree in high voltage engineering from the Indian Institute of Science, Bangalore India in 2000. He worked as a Materials Scientist in the area of electrical insulation systems at the General Electrical Global Research Center in Bangalore, India during 2000-2003. Since 2004, he has been a graduate student at the Indian Institute of Science, Bangalore, India working towards his Ph.D. degree in dielectrics and electrical insulation technology. His areas of interest are in dielectric nanocomposites, gas insulation systems, modeling and design of transformer insulation systems and design of electrical insulation systems for HV equipment.

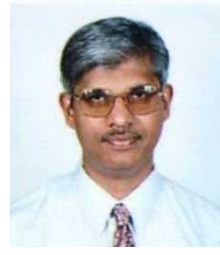

M. Joy Thomas (S'85-M'95) was born in Kerala, India in 1961. He received the B.Tech. degree in electrical engineering from Institute of Technology, BHU, Varanasi, India in 1983, the M.Sc. (Engg.) and Ph.D. degrees from Indian Institute of Science, Bangalore, India. Presently, he is an Assistant Professor at the High Voltage Laboratory, Department of Electrical Engineering, Indian Institute of Science, Bangalore, India. $\mathrm{He}$ is also a member of CIGRE and ASEE. His areas of interest are EHV/UHV power transmission, high voltage engineering, dielectrics and electrical insulation, pulsed power engineering, plasma science and technology, EMC and engineering pedagogy. 